\title{
On a more accurate half-discrete Hilbert's inequality
}

Qiliang Huang ${ }^{*}$ and Bicheng Yang

* Correspondence: qlhuang@yeah. net

Department of Mathematics, Guangdong University of Education, Guangzhou, Guangdong 510303, People's Republic of China

\section{Abstract}

By using the way of weight coefficients and the idea of introducing parameters and by means of Hadamard's inequality, we give a more accurate half-discrete Hilbert's inequality with a best constant factor. We also consider its best extension with parameters, the equivalent forms, the operator expressions as well as some reverses. 2000 Mathematics Subject Classification: 26D15; $47 \mathrm{~A} 07$.

Keywords: weight coefficient, parameter, equivalent form, reverse, Hilbert's inequality, Hadamard's inequality

\section{Introduction}

If $a_{n}, b_{n} \geq 0,0<\sum_{n=1}^{\infty} a_{n}^{2}<\infty$ and $0<\sum_{n=1}^{\infty} b_{n}^{2}<\infty$, then we have the following well-known Hilbert's inequality (cf. [1]):

$$
\sum_{n=1}^{\infty} \sum_{m=1}^{\infty} \frac{a_{m} b_{n}}{m+n}<\pi\left(\sum_{m=1}^{\infty} a_{m}^{2} \sum_{n=1}^{\infty} b_{n}^{2}\right)^{1 / 2}
$$

where the constant factor $\pi$ is the best possible. The integral analogue of inequality (1) is given as follows (cf. [2]): If $0<\int_{0}^{\infty} f^{2}(x) d x<\infty$ and $0<\int_{0}^{\infty} g^{2}(x) d x<\infty$, then

$$
\int_{0}^{\infty} \int_{0}^{\infty} \frac{f(x) g(y)}{x+y} d x d y<\pi\left(\int_{0}^{\infty} f^{2}(x) d x \int_{0}^{\infty} g^{2}(x) d x\right)^{1 / 2}
$$

where the constant factor $\pi$ is the best possible. We named inequality (2) as Hilbert's integral inequality. Hardy et al. [3] proved the following more accurate Hilbert's inequality:

$$
\sum_{n=1}^{\infty} \sum_{m=1}^{\infty} \frac{a_{m} b_{n}}{m+n-1}<\pi\left(\sum_{m=1}^{\infty} a_{m}^{2} \sum_{n=1}^{\infty} b_{n}^{2}\right)^{1 / 2},
$$

where the constant factor $\pi$ is still the best possible. Inequalities (1)-(3) are important in analysis and its applications [4]. There are lots of improvements, generalizations, and applications of inequalities (1-3), for more details, refer to literatures [5-18].

(C) 2012 Huang and Yang; licensee Springer. This is an Open Access article distributed under the terms of the Creative Commons Attribution License (http://creativecommons.org/licenses/by/2.0), which permits unrestricted use, distribution, and reproduction in any medium, provided the original work is properly cited. 
We find a few results on the half-discrete Hilbert-type inequalities with the nonhomogeneous kernel, which were published early ([[3], Th. 351], [19]). Recently, Yang [20-22] gave some half-discrete Hilbert-type inequalities. A half-discrete Hilbert's inequality with the homogeneous kernel was derived as follows [20]: If $0<\int_{0}^{\infty} f^{2}(x) d x<\infty$ and $0<\sum_{n=1}^{\infty} a_{n}^{2}<\infty$, then

$$
\sum_{n=1}^{\infty} a_{n} \int_{0}^{\infty} \frac{f(x)}{x+n} d x<\pi\left(\sum_{n=1}^{\infty} a_{n}^{2} \int_{0}^{\infty} f^{2}(x) d x\right)^{1 / 2},
$$

where the constant factor $\pi$ is the best possible.

In this article, by using the way of weight coefficients and the idea of introducing parameters and by means of Hadamard's inequality, we give a more accurate inequality of (4) with a best constant factor as follows:

$$
\sum_{n=1}^{\infty} a_{n} \int_{-\frac{1}{2}}^{\infty} \frac{f(x)}{x+n} d x<\pi\left(\sum_{n=1}^{\infty} a_{n}^{2} \int_{-\frac{1}{2}}^{\infty} f^{2}(x) d x\right)^{1 / 2}
$$

We also consider its best extension with parameters, the equivalent forms, the operator expressions as well as some reverses.

\section{Some lemmas}

Lemma 1 Suppose $0<\alpha \leq 1,0 \leq \beta \leq \frac{1}{2}, \gamma \in(-\infty, \infty), \lambda_{1}>0,0<\lambda_{2} \alpha \leq 1, \lambda=\lambda_{1}+\lambda_{2}$. Define the beta function (cf. [18]) and the weight coefficients as follows:

$$
\begin{aligned}
& B(u, v):=\int_{0}^{\infty} \frac{t^{u-1} d t}{(1+t)^{u+v}}=\int_{0}^{1} \frac{t^{u-1}+t^{\nu-1}}{(1+t)^{u+v} d t(u, v>0),} \\
& \omega(n):=(n-\beta)^{\lambda_{2} \alpha} \int_{\gamma}^{\infty} \frac{(x-\gamma)^{\lambda_{1} \alpha-1}}{\left[(x-\gamma)^{\alpha}+(n-\beta)^{\alpha}\right]^{\lambda}} d x(n \in \mathrm{N}), \\
& \varpi(x):=(x-\gamma)^{\lambda_{1} \alpha} \sum_{n=1}^{\infty} \frac{(n-\beta)^{\lambda_{2} \alpha-1}}{\left[(x-\gamma)^{\alpha}+(n-\beta)^{\alpha}\right]^{\lambda}}(x \in(\gamma, \infty)) .
\end{aligned}
$$

Setting $k_{\lambda_{1}}(\alpha):=\frac{1}{\alpha} B\left(\lambda_{1}, \lambda_{2}\right)$, we have the following inequalities:

$$
0<k_{\lambda_{1}}(\alpha)\left(1-\theta_{\lambda}(x)\right)<\varpi(x)<\omega(n)=k_{\lambda_{1}}(\alpha),
$$

where, $\theta_{\lambda}(x):=\frac{1}{B\left(\lambda_{1}, \lambda_{2}\right)} \int_{0}^{\left(\frac{1-\beta}{x-\gamma}\right)^{\alpha}} \frac{u^{\lambda_{2}-1}}{(1+u)^{\lambda}} d u>0$ and $\theta_{\lambda}(x)=O\left(\frac{1}{(x-\gamma)^{\lambda_{2} \alpha}}\right)(x \in(\gamma, \infty))$. 
Proof. Putting $u=\left(\frac{x-\gamma}{n-\beta}\right)^{\alpha}$ in (7), we have

$$
\omega(n)=\frac{1}{\alpha} \int_{0}^{\infty} \frac{u^{\lambda_{1}-1}}{(1+u)^{\lambda}} d u=\frac{1}{\alpha} B\left(\lambda_{1}, \lambda_{2}\right)=k_{\lambda_{1}}(\alpha) .
$$

For fixed $x \in(\gamma, \infty)$, setting

$$
f(t):=\frac{(x-\gamma)^{\lambda_{1} \alpha}(t-\beta)^{\lambda_{2} \alpha-1}}{\left[(x-\gamma)^{\alpha}+(t-\beta)^{\alpha}\right]^{\lambda}}(t \in(\beta, \infty)),
$$

in view of the conditions, we find $f^{\prime}(t)<0$ and $f^{\prime \prime}(t)>0$. By the following Hadamard's inequality (cf. [18]):

$$
f(n)<\int_{n-\frac{1}{2}}^{n+\frac{1}{2}} f(t) d t(n \in \mathrm{N})
$$

and putting $u=\left(\frac{t-\beta}{x-\gamma}\right)^{\alpha}$, it follows

$$
\begin{aligned}
\varpi(x) & =\sum_{n=1}^{\infty} f(n)<\sum_{n=1}^{\infty} \int_{n-\frac{1}{2}}^{n+\frac{1}{2}} f(t) d t=\int_{\frac{1}{2}}^{\infty} f(t) d t \\
& <\int_{\beta}^{\infty} f(t) d t=\frac{1}{\alpha} \int_{0}^{\infty} \frac{u^{\lambda_{2}-1}}{(1+u)^{\lambda}} d u=\frac{1}{\alpha} B\left(\lambda_{2}, \lambda_{1}\right)=k_{\lambda_{1}}(\alpha), \\
\varpi(x) & =\sum_{n=1}^{\infty} f(n)>\int_{1}^{\infty} f(t) d t=\int_{\beta}^{\infty} f(t) d t-\int_{\beta}^{1} f(t) d t \\
& =k_{\lambda_{1}}(\alpha)-\frac{1}{\alpha} \int_{0}^{\left(\frac{1-\beta}{x-\gamma}\right)^{\alpha}} \frac{u^{\lambda_{2}-1}}{(1+u)^{\lambda}} d u=k_{\lambda_{1}}(\alpha)\left(1-\theta_{\lambda}(x)\right)>0,
\end{aligned}
$$

where

$$
\begin{aligned}
0<\theta_{\lambda}(x) & =\frac{1}{B\left(\lambda_{1}, \lambda_{2}\right)} \int_{0}^{\left(\frac{1-\beta}{x-\gamma}\right)^{\alpha}} \frac{u^{\lambda_{2}-1}}{(1+u)^{\lambda}} d u \\
& <\frac{1}{B\left(\lambda_{1}, \lambda_{2}\right)} \int_{0}^{\left(\frac{1-\beta}{x-\gamma}\right)^{\alpha}} u^{\lambda_{2}-1} d u=\frac{(1-\beta)^{\lambda_{2} \alpha}}{\lambda_{2} B\left(\lambda_{1}, \lambda_{2}\right)} \frac{1}{(x-\gamma)^{\lambda_{2} \alpha}} .
\end{aligned}
$$

Hence, we prove that (9) is valid.

Lemma 2 Suppose that $\frac{1}{p}+\frac{1}{q}=1(p \neq 0,1), 0<\alpha \leq 1,0 \leq \beta \leq \frac{1}{2}, \gamma \in(-\infty+\infty), \lambda_{1}>$ $0,0<\lambda_{2} \alpha \leq 1, \lambda=\lambda_{1}+\lambda_{2}, \alpha_{n} \geq 0, f(x) \geq 0$ is a real measurable function in $(\gamma, \infty)$, then (i) for $p>1$, we have the following 


$$
\begin{aligned}
J & :=\left\{\sum_{n=1}^{\infty}(n-\beta)^{p \lambda_{2} \alpha-1}\left[\int_{\gamma}^{\infty} \frac{f(x)}{\left[(x-\gamma)^{\alpha}+(n-\beta)^{\alpha}\right]^{\lambda}} d x\right]^{p}\right\}^{1 / p} \\
& \leq\left(k_{\lambda_{1}}(\alpha)\right)^{1 / q}\left\{\int_{\gamma}^{\infty} \varpi(x)(x-\gamma)^{p\left(1-\lambda_{1} \alpha\right)-1} f^{p}(x) d x\right\}^{1 / p}, \\
L_{1} & :=\left\{\int_{\gamma}^{\infty} \frac{(x-\gamma)^{q \lambda_{1} \alpha-1}}{\varpi^{q-1}(x)}\left[\sum_{n=1}^{\infty} \frac{a_{n}}{\left[(x-\gamma)^{\alpha}+(n-\beta)^{\alpha}\right]^{\lambda}}\right]^{q} d x\right\}^{1 / q} \\
& <\left\{k_{\lambda_{1}}(\alpha) \sum_{n=1}^{\infty}(n-\beta)^{q\left(1-\lambda_{2} \alpha\right)-1} a_{n}^{q}\right\}^{1 / q},
\end{aligned}
$$

where $\omega(x)$ and $\omega(n)$ are indicated by (7) and (8).

(ii) for $p<1(p \neq 0)$, we have the reverses of (13) and (14).

Proof. (i) By (7)-(9) and Hölder's inequality (cf. [18]), we find

$$
\begin{aligned}
& {\left[\int_{\gamma}^{\infty} \frac{f(x)}{\left[(x-\gamma)^{\alpha}+(n-\beta)^{\alpha}\right]^{\lambda}} d x\right]^{p}=\left\{\int_{\gamma}^{\infty} \frac{1}{\left[(x-\gamma)^{\alpha}+(n-\beta)^{\alpha}\right]^{\lambda}}\right.} \\
& \times\left[\frac{(x-\gamma)^{\left(1-\lambda_{1} \alpha\right) / q}}{(n-\beta)^{\left(1-\lambda_{2} \alpha\right) / q}} f(x)\right]\left[\frac{(n-\beta)^{\left(1-\lambda_{2} \alpha\right) / q}}{(x-\gamma)^{\left(1-\lambda_{1} \alpha\right) / q}} d x\right]^{p} \\
\leq & \int_{\gamma}^{\infty} \frac{1}{\left[(x-\gamma)^{\alpha}+(n-\beta)^{\alpha}\right]^{\lambda}} \frac{(x-\gamma)^{\left(1-\lambda_{1} \alpha\right)(\rho-1)}}{(n-\beta)^{1-\lambda_{2} \alpha}} f^{p}(x) d x \\
& \times\left[\int_{\gamma}^{\infty} \frac{1}{\left[(x-\gamma)^{\alpha}+(n-\beta)^{\alpha}\right]^{\lambda}} \frac{(n-\beta)^{\left(1-\lambda_{2} \alpha\right)(q-1)}}{(x-\gamma)^{1-\lambda_{1} \alpha}} d x\right]^{p-1} \\
= & \int_{\gamma}^{\infty} \frac{f^{p}(x)(x-\gamma)^{\left(1-\lambda_{1} \alpha\right)(p-1)}}{\left[(x-\gamma)^{\alpha}+(n-\beta)^{\alpha}\right]^{\lambda}} \frac{d x}{(n-\beta)^{1-\lambda_{2} \alpha}}\left[(n-\beta)^{q\left(1-\lambda_{2} \alpha\right)-1} \omega(n)\right]^{p-1} \\
= & (n-\beta)^{1-p \lambda_{2} \alpha} k_{\lambda_{1}}^{p-1}(\alpha) \int_{\gamma}^{\infty} \frac{f^{p}(x)(x-\gamma)^{\left(1-\lambda_{1} \alpha\right)(\rho-1)}}{\left[(x-\gamma)^{\alpha}+(n-\beta)^{\alpha}\right]^{\lambda}} \frac{1}{(n-\beta)^{1-\lambda_{2} \alpha}} d x, \\
= & k_{\lambda_{1}}^{p-1}(\alpha) \int_{\gamma}^{\infty} \varpi(x)(x-\gamma)^{p\left(1-\lambda_{1} \alpha\right)-1} f^{p}(x) d x . \\
= & k_{\lambda_{1}}^{p-1}(\alpha) k_{\gamma}^{p-1} \sum_{n=1}^{\infty} \frac{(n) \sum_{n=1}^{\infty} \int_{\gamma}^{\infty} \frac{(n-\beta)^{\lambda_{2} \alpha-1}}{\left[(x-\gamma)^{\alpha}+(n-\beta)^{\alpha}\right]^{\lambda}}(x-\gamma)^{\lambda_{1} \alpha+p\left(1-\lambda_{1} \alpha\right)-1} f^{p}(x) d x}{\left.(n-\beta)^{\alpha}\right]^{\lambda}} \frac{(x-\gamma)^{\left(1-\lambda_{1} \alpha\right)(p-1)}}{(n-\beta)^{1-\lambda_{2} \alpha}} d x \\
& {[(x)} \\
&
\end{aligned}
$$


Hence (13) is valid. Using Hölder's inequality again, we have

$$
\begin{aligned}
& {\left[\sum_{n=1}^{\infty} \frac{a_{n}}{\left[(x-\gamma)^{\alpha}+(n-\beta)^{\alpha}\right]^{\lambda}}\right]^{q}=\left\{\sum_{n=1}^{\infty} \frac{1}{\left[(x-\gamma)^{\alpha}+(n-\beta)^{\alpha}\right]^{\lambda}}\right.} \\
& \left.\times\left[\frac{(x-\gamma)^{\left(1-\lambda_{1} \alpha\right) / q}}{(n-\beta)^{\left(1-\lambda_{2} \alpha\right) / q}}\right]\left[\frac{(n-\beta)^{\left(1-\lambda_{2} \alpha\right) / q}}{(x-\gamma)^{\left(1-\lambda_{1} \alpha\right) / q}} a_{n}\right]\right\}^{q} \\
& \leq\left[\varpi(x)(x-\gamma)^{p\left(1-\lambda_{1} \alpha\right)-1}\right]^{q-1} \sum_{n=1}^{\infty} \frac{a_{n}^{q}}{\left[(x-\gamma)^{\alpha}+(n-\beta)^{\alpha}\right]^{\lambda}} \frac{(n-\beta)^{\left(1-\lambda_{2} \alpha\right) q / p}}{(x-\gamma)^{1-\lambda_{1} \alpha}} \\
& =\varpi^{q-1}(x)(x-\gamma)^{1-q \lambda_{1} \alpha} \sum_{n=1}^{\infty} \frac{(n-\beta)^{\left(1-\lambda_{2} \alpha\right)(q-1) \mid}}{\left[(x-\gamma)^{\alpha}+(n-\beta)^{\alpha}\right]^{\lambda}} \frac{1}{(x-\gamma)^{1-\lambda_{1} \alpha}} a_{n}^{q}, \\
& L_{1}^{q} \leq \int_{\gamma}^{\infty} \sum_{n=1}^{\infty} \frac{1}{\left[(x-\gamma)^{\alpha}+(n-\beta)^{\alpha}\right]^{\lambda}} \frac{(n-\beta)^{\left(1-\lambda_{2} \alpha\right)(q-1)}}{(x-\gamma)^{1-\lambda_{1} \alpha}} a_{n}^{q} d x \\
& =\sum_{n=1}^{\infty}\left[(n-\beta)^{\lambda_{2} \alpha} \int_{\gamma}^{\infty} \frac{(x-\gamma)^{\lambda_{1} \alpha-1}}{\left[(x-\gamma)^{\alpha}+(n-\beta)^{\alpha}\right]^{\lambda}} d x\right](n-\beta)^{q\left(1-\lambda_{2} \alpha\right)-1} a_{n}^{q} \\
& =\sum_{n=1}^{\infty} \omega(n)(n-\beta)^{q\left(1-\lambda_{2} \alpha\right)-1} a_{n}^{q}=k_{\lambda_{1}}(\alpha) \sum_{n=1}^{\infty}(n-\beta)^{q(1-\lambda 2 \alpha)-1} a_{n}^{q} .
\end{aligned}
$$

Hence (14) is valid.

(ii) For $0<p<1(q<0)$ or $p<0(0<q<1)$, using the reverse Hölder's inequality and in the same way, we have the reverses of (13) and (14).

Lemma 3 As the assumptions of Lemmas 1 and 2, we set $\phi(x):=(x-\gamma)^{p\left(1-\lambda_{1} \alpha\right)-1}$,

$$
\begin{aligned}
& \psi(n):=(n-\beta)^{q\left(1-\lambda_{2} \alpha\right)-1}, \psi(n):=(n-\beta)^{q\left(1-\lambda_{2} \alpha\right)-1}, \\
& L_{p, \phi}(\gamma, \infty):=\left\{f ;\|f\|_{p, \phi}=\left\{\int_{\gamma}^{\infty} \phi(x)|f(x)|^{p} d x\right\}^{1 / p}<\infty\right\}, \\
& l_{q, \psi}:=\left\{a=\left\{a_{n}\right\} ;\|a\|, \psi=\left\{\sum_{n=1}^{\infty} \psi(n)\left|a_{n}\right|^{q}\right\}^{1 / q}<\infty\right\}
\end{aligned}
$$

(Note. if $p>1$, then $L_{p, \varphi}(\gamma, \infty)$ and $l_{q, \psi}$ are normal spaces; if $0<p<1$ or $p<0$, then both $L_{p}, \varphi(\gamma, \infty)$ and $l_{q, \psi}$ are not normal spaces, but we still use the formal symbols in the following.) For $0<\varepsilon<\min \left\{1, \lambda_{1} p \alpha\right\}$, setting $\tilde{a}=\left\{\tilde{a}_{n}\right\}_{n=1}^{\infty}$ and $\tilde{f}(x)$ as follows

$$
\tilde{a}_{n}=(n-\beta)^{\lambda_{2} \alpha-\frac{\varepsilon}{q}-1} ; \tilde{f}(x)= \begin{cases}0, & x \in(\gamma, 1+\gamma), \\ (x-\gamma)^{\lambda_{1} \alpha-\frac{\varepsilon}{p}-1}, & x \in[1+\gamma, \infty),\end{cases}
$$

(i) if $p>1$, there exists a constant $k>0$, such that

$$
\tilde{I}:=\sum_{n=1}^{\infty} \tilde{a}_{n} \int_{\gamma}^{\infty} \frac{\tilde{f}(x)}{\left[(x-\gamma)^{\alpha}+(n-\beta)^{\alpha}\right]^{\lambda}} d x<k\|\tilde{f}\|_{p, \phi}\|\tilde{a}\|_{q, \psi^{\prime}}
$$


then it follows

$$
k\left(\frac{\varepsilon+1-\beta}{(1-\beta)^{\varepsilon+1}}\right)^{1 / q} \geq \frac{1}{\alpha} B\left(\lambda_{2}+\frac{\varepsilon}{p \alpha}, \lambda_{1}+\frac{\varepsilon}{p \alpha}\right)-\varepsilon O(1)
$$

(ii) if $0<p<1$, there exists a constant $k>0$, such that

$$
\tilde{I}-\sum_{n=1}^{\infty} \tilde{a}_{n} \int_{\gamma}^{\infty} \frac{\tilde{f}(x)}{\left[(x-\gamma)^{\alpha}+(n-\beta)^{\alpha}\right]^{\lambda}} d x>k\|\tilde{f}\|_{p, \tilde{\phi}}\|\tilde{a}\|_{q, \psi^{\prime}}
$$

then it follows

$$
k(1-\varepsilon O(1))^{1 / p}<\frac{1}{\alpha}\left(\frac{\varepsilon+1-\beta}{(1-\beta)^{\varepsilon+1}}\right)^{1 / p} B\left(\lambda_{1}-\frac{\varepsilon}{p \alpha}, \lambda_{2}+\frac{\varepsilon}{p \alpha}\right) .
$$

Proof. we obtain

$$
\begin{aligned}
\|\tilde{f}\|_{p, \phi} & =\left\{\int_{\gamma}^{\infty}(x-\gamma)^{p\left(1-\lambda_{1} \alpha\right)-1} \tilde{f}^{p}(x) d x\right\}^{1 / p} \\
& =\left\{\int_{1+\gamma}^{\infty}(x-\gamma)^{-1-\varepsilon} d x\right\}^{1 / p}=\left(\frac{1}{\varepsilon}\right)^{1 / p}, \\
\|\tilde{a}\|_{q, \psi}^{q} & =\sum_{n=1}^{\infty}(n-\beta)^{q\left(1-\lambda_{2} \alpha\right)-1} \tilde{a}_{n}^{q}=\sum_{n=1}^{\infty}(n-\beta)^{-1-\varepsilon} \\
& <(1-\beta)^{-1-\varepsilon}+\int_{1}^{\infty}(x-\beta)^{-1-\varepsilon} d x=\frac{\varepsilon+1-\beta}{\varepsilon(1-\beta)^{\varepsilon+1}} .
\end{aligned}
$$

(i) For $p>1$, then $q>1, \lambda_{2} \alpha-\frac{\varepsilon}{q}-1<0$, by (20), (24), and (25), we find

$$
\begin{aligned}
\tilde{I} & <k\left(\frac{1}{\varepsilon}\right)^{1 / p}\left[\frac{\varepsilon+1-\beta}{\varepsilon(1-\beta)^{\varepsilon+1}}\right]^{1 / q}=\frac{k}{\varepsilon}\left[\frac{\varepsilon+1-\beta}{(1-\beta)^{\varepsilon+1}}\right]^{1 / q}, \\
\tilde{I} & =\int_{1+\gamma}^{\infty}(x-\gamma)^{\lambda_{1} \alpha-\frac{\varepsilon}{p}-1}\left(\sum_{n=1}^{\infty} \frac{(n-\beta)^{\lambda_{2} \alpha-\frac{\varepsilon}{q}-1}}{\left[(x-\gamma)^{\alpha}+(n-\beta)^{\alpha}\right]^{\lambda}}\right) d x \\
& \geq \int_{1+\gamma}^{\infty}(x-\gamma)^{\lambda_{1} \alpha-\frac{\varepsilon}{p}-1}\left(\int_{1}^{\infty} \frac{(\gamma-\beta)^{\lambda_{2} \alpha-\frac{\varepsilon}{q}-1}}{\left[(x-\gamma)^{\alpha}+(\gamma-\beta)^{\alpha}\right]^{\lambda}} d \gamma\right) d x .
\end{aligned}
$$

Setting $s=x-\gamma, t=\left(\frac{\gamma-\beta}{x-\gamma}\right)^{\alpha}$ in the above integral, we have

$$
\tilde{I} \geq \frac{1}{\alpha} \int_{1}^{\infty} s^{-1-\varepsilon}\left[\int_{\left(\frac{1-\beta}{s}\right)^{\varepsilon}}^{\infty} t^{\lambda_{2}-\frac{\varepsilon}{q \alpha}-1} \frac{1}{(1+t)^{\lambda}} d t\right] d s=A+B
$$


where

$$
\begin{aligned}
A: & =\frac{1}{\alpha} \int_{1}^{\infty} s^{-1-\varepsilon} \int_{\left(\frac{1-\beta}{s}\right)^{\alpha}}^{1} \frac{t^{\lambda_{2}-\frac{\varepsilon}{q \alpha}-1}}{(1+t)^{\lambda}} d t d s ; \\
B: & =\frac{1}{\alpha} \int_{1}^{\infty} s^{-1-\varepsilon} \int_{1}^{\infty} \frac{t^{\lambda_{2}-\frac{\varepsilon}{q \alpha}-1}}{(1+t)^{\lambda}} d t d s=\frac{1}{\alpha \varepsilon} \int_{1}^{\infty} \frac{t^{\lambda_{2}-\frac{\varepsilon}{q \alpha}-1}}{(1+t)^{\lambda}} d t \\
& u=\frac{1}{t} \frac{1}{\alpha \varepsilon} \int_{0}^{1} \frac{u^{\lambda_{1}+\frac{\varepsilon}{q \alpha}-1}}{(1+u)^{\lambda}} d u \geq \frac{1}{\alpha \varepsilon} \int_{0}^{1} \frac{u^{\lambda_{1}+\frac{\varepsilon}{q \alpha}-1}}{(1+u)^{\lambda+\varepsilon / \alpha}} d u .
\end{aligned}
$$

Since

$$
0<\frac{1}{\alpha} \int_{1-\beta}^{1} s^{-1-\varepsilon} \int_{\left(\frac{1-\beta}{s}\right)}^{1} \frac{t^{\lambda_{2}-\frac{\varepsilon}{q \alpha}}-1}{(1+t)^{\lambda}} d t d s \leq \frac{1}{\alpha} \int_{1-\beta}^{1} \int_{(1-\beta)^{\alpha}}^{1} s^{-2} \frac{t^{\lambda_{2}-\frac{1}{\alpha}-1}}{(1+t)^{\lambda}} d t d s<\infty,
$$

then by Fubini's theorem, we have

$$
\begin{aligned}
A & =\frac{1}{\alpha}\left[\int_{1-\beta}^{\infty} s^{-1-\varepsilon} \int_{\left(\frac{1-\beta}{s}\right)^{\alpha}}^{1} \frac{t^{\lambda_{2}-\frac{\varepsilon}{q \alpha}-1}}{(1+t)^{\lambda}} d t d s-\int_{1-\beta}^{1} s^{-1-\varepsilon} \int_{\left(\frac{1-\beta}{s}\right)^{\alpha}}^{1} \frac{t^{\lambda_{2}-\frac{\varepsilon}{q \alpha}-1}}{(1+t)^{\lambda}} d t d s\right] \\
& =\frac{1}{\alpha} \int_{0}^{1} \frac{t^{\lambda_{2}-\frac{\varepsilon}{q \alpha}-1}}{(1+t)^{\lambda}}\left[\int_{(1-\beta) / t^{(1 / \alpha)}}^{\infty} s^{-1-\varepsilon} d s\right] d t-O(1) \\
& =\frac{(1-\beta)^{-\varepsilon}}{\alpha \varepsilon} \int_{0}^{1} \frac{t^{\lambda_{2}+\frac{\varepsilon}{q \alpha}-1}}{(1+t)^{\lambda}} d t-O(1) \geq \frac{1}{\alpha \varepsilon} \int_{0}^{1} \frac{t^{\lambda_{2}+\frac{\varepsilon}{q \alpha}-1}}{(1+t)^{\lambda+\varepsilon / \alpha}} d t-O(1) .
\end{aligned}
$$

In view of (28) and (29) and (6), it follows that

$$
A+B \geq \frac{1}{\alpha \varepsilon} \int_{0}^{1} \frac{t^{\lambda_{1}+\frac{\varepsilon}{q \alpha}-1}+t^{\lambda_{2}+\frac{\varepsilon}{q \alpha}-1}}{(1+t)^{\lambda+\varepsilon / \alpha}} d t-O(1)=\frac{1}{\alpha \varepsilon} B\left(\lambda_{2}+\frac{\varepsilon}{p \alpha}, \lambda_{1}+\frac{\varepsilon}{q \alpha}\right)-O(1) .
$$

Then by (26) and (27), (21) is valid.

(ii) For $0<p<1$, by (22) and (25), we find (notice that $q<0$ )

$$
\begin{aligned}
\tilde{I} & >k\left\{\int_{1+\gamma}^{\infty}\left[1-O\left(\frac{1}{(x-\gamma)^{\lambda_{2} \alpha}}\right)\right](x-\gamma)^{-1-\varepsilon} d x\right\}^{1 / p}\|\tilde{a}\|_{\mathrm{q}, \psi} \\
& =k\left(\frac{1}{\varepsilon}-\int_{1+\gamma}^{\infty} O\left(\frac{1}{(x-\gamma)^{\lambda_{2}^{\alpha+\varepsilon+1}}}\right) d x\right)^{1 / \mathrm{p}}\|\tilde{a}\|_{q, \psi} \\
> & k\left(\frac{1}{\varepsilon}-O(1)\right)^{1 / p}\left(\frac{\varepsilon+1-\beta}{\varepsilon(1-\beta)^{\varepsilon+1}}\right)^{1 / q} \\
& =\frac{k}{\varepsilon}(1-\varepsilon O(1))^{1 / p}\left(\frac{\varepsilon+1-\beta}{(1-\beta)^{\varepsilon+1}}\right)^{1 / q} .
\end{aligned}
$$


On the other hand, setting $t=\left(\frac{x-\gamma}{n-\beta}\right)^{\alpha}$ in $\tilde{I}$, we have

$$
\begin{aligned}
\tilde{I} & =\sum_{n=1}^{\infty}(n-\beta)^{-1-\varepsilon} \frac{1}{\alpha} \int_{1 /(n-\beta)^{\alpha}}^{\infty} \frac{t^{\lambda_{1}-\frac{\varepsilon}{p \alpha}-1}}{(1+t)^{\lambda}} d t \\
& \leq \sum_{n=1}^{\infty}(n-\beta)^{-1-\varepsilon} \frac{1}{\alpha} \int_{0}^{\infty} \frac{t^{\lambda_{1}-\frac{\varepsilon}{p \alpha}-1}}{(1+t)^{\lambda}} d t \\
& <\frac{\varepsilon+1-\beta}{\alpha \varepsilon(1-\beta)^{\varepsilon+1}} B\left(\lambda_{1}-\frac{\varepsilon}{p \alpha}, \lambda_{2}+\frac{\varepsilon}{p \alpha}\right) .
\end{aligned}
$$

In virtue of (30) and (31), (23) is valid.

\section{Main results}

Theorem 1 Suppose that $p>1, \frac{1}{p}+\frac{1}{q}=1,0<\alpha \leq 1,0 \leq \beta \leq \frac{1}{2}, \gamma \in(-\infty,+\infty), \lambda_{1}>0$, $0<\lambda_{2} \alpha \leq 1, \lambda=\lambda_{1}+\lambda_{2}, \phi(x)=(x-\gamma)^{p\left(1-\lambda_{1} \alpha\right)-1}, \psi(n)=(n-\beta)^{q\left(1-\lambda_{2} \alpha\right)-1}, f(x), a_{n} \geq$ 0 , such that $f \in L_{p, \varphi}(\gamma, \infty), a=\left\{a_{n}\right\}_{n=1}^{\infty} \in l_{q, \psi},\|f\|_{p, \varphi}>0,\|a\|_{q, \psi}>0$, then we have the following equivalent inequalities:

$$
\begin{aligned}
I & :=\sum_{n=1}^{\infty} a_{n} \int_{\gamma}^{\infty} \frac{f(x)}{\left[(x-\gamma)^{\alpha}+(n-\beta)^{\alpha}\right]^{\lambda}} d x \\
& =\int_{\gamma}^{\infty} f(x) \sum_{n=1}^{\infty} \frac{a_{n} d x}{\left[(x-\gamma)^{\alpha}+(n-\beta)^{\alpha}\right]^{\lambda}}<k_{\lambda_{1}}(\alpha)\|f\|_{p, \phi}\|a\|_{q, \psi^{\prime}} \\
J & =\left\{\sum_{n=1}^{\infty}(n-\beta)^{p \lambda_{2} \alpha-1}\left[\int_{\gamma}^{\infty} \frac{f(x) d x}{\left[(x-\gamma)^{\alpha}+(n-\beta)^{\alpha}\right]^{\lambda}}\right]^{p}\right\}^{\frac{1}{p}}<k_{\lambda_{1}}(\alpha)\|f\|_{p, \phi^{\prime}} \\
L & =\left\{\int_{\gamma}^{\infty}(x-\gamma)^{q \lambda_{1} \alpha-1}\left[\sum_{n=1}^{\infty} \frac{a_{n}}{\left[(x-\gamma)^{\alpha}+(n-\beta)^{\alpha}\right]^{\lambda}}\right]^{q} d x\right\}^{\frac{1}{q}<k_{\lambda_{1}}(\alpha)\|a\|_{q, \psi},}
\end{aligned}
$$

where the constant factor $k_{\lambda_{1}}(\alpha)=\frac{1}{\alpha} B\left(\lambda_{1}, \lambda_{2}\right)$ is the best possible.

Proof. By Lebesgue term-by-term integration theorem [23], we find that there are two expressions of $I$ in (32). By (9), (13) and $0<\left.|| f\right|_{p, \varphi}<\infty$, we have (33). By Hölder's inequality, we find

$$
\begin{aligned}
I & =\sum_{n=1}^{\infty}\left[(n-\beta)^{\lambda_{2} \alpha-\frac{1}{p}} \int_{\gamma}^{\infty} \frac{f(x) d x}{\left[(x-\gamma)^{\alpha}+(n-\beta)^{\alpha}\right]^{\lambda}}\right]\left[(n-\beta)^{\frac{1}{p}-\lambda_{2} \alpha} a_{n}\right] \\
& \leq J\left\{\sum_{n=1}^{\infty}(n-\beta)^{q\left(1-\lambda_{2} \alpha\right)-1} a_{n}^{q}\right\}^{1 / q}=J\|a\|_{q, \psi} .
\end{aligned}
$$

Hence (32) is valid by (33). On the other hand, setting

$$
a_{n}:=(n-\beta)^{p \lambda_{2} \alpha-1}\left[\int_{\gamma}^{\infty} \frac{f(x)}{\left[(x-\gamma)^{\alpha}+(n-\beta)^{\alpha}\right]^{\lambda}} d x\right]^{p-1} \quad(n \in \mathbf{N}) \text {, }
$$


then we have

$$
\|a\|_{q, \psi}^{q}=\sum_{n=1}^{\infty}(n-\beta)^{q\left(1-\lambda_{2} \alpha\right)-1} a_{n}^{q}=J^{p}=I .
$$

By (9), (13) and $0<|| f||_{p, \varphi}<\infty$, it follows that $J<\infty$. If $J=0$, then (33) is trivially valid. If $J>0$, then $0<\|\left. a\right|_{q, \psi}=J^{p-1}<\infty$. Assuming that (32) is valid, we have

$$
\|a\|_{q, \psi}^{q}=J^{p}=I<k_{\lambda_{1}}(\alpha)\|f\|_{p, \phi}\|a\|_{q, \psi} \text {, i.e. } J=\|a\|_{q, \psi}^{q-1}<k_{\lambda_{1}}(\alpha)\|f\|_{p, \phi} .
$$

Hence (33) is valid, which is equivalent to (32).

By (14) and (9), we obtain (34). By Hölder's inequality again, we have

$$
\begin{aligned}
I & =\int_{\gamma}^{\infty}\left[(x-\gamma)^{\lambda_{1} \alpha-\frac{1}{q}} \sum_{n=1}^{\infty} \frac{a_{n}}{\left[(x-\gamma)^{\alpha}+(n-\beta)^{\alpha}\right]^{\lambda}}\right]\left[(x-\gamma)^{\frac{1}{q}-\lambda_{1} \alpha} f(x)\right] d x \\
& \leq L\left\{\int_{\gamma}^{\infty}(x-\gamma)^{p\left(1-\lambda_{1} \alpha\right)-1} f^{p}(x) d x\right\}^{1 / p}=L\|f\|_{p, \phi} .
\end{aligned}
$$

Hence (32) is valid by using (34). Assuming that (32) is valid, setting

$$
f(x):=(x-\gamma)^{q \lambda_{1} \alpha-1}\left[\sum_{n=1}^{\infty} \frac{a_{n}}{\left[(x-\gamma)^{\alpha}+(n-\beta)\right]^{\lambda}}\right]^{q-1}(x \in(\gamma, \infty)),
$$

then we find

$$
\|f\|_{p, \phi}^{p}=\int_{\gamma}^{\infty}(x-\gamma)^{p\left(1-\lambda_{1} \alpha\right)-1} f^{p}(x) d x=L^{q}=I .
$$

By (14) and (9), it follows that $L<\infty$. If $L=0$, then (34) is trivially valid; if $L>0$, i.e. $0<\|f\|_{p, \varphi}<\infty$, then by (32), we have

$$
\|f\|_{p, \phi}^{p}=L^{q}=I<k_{\lambda_{1}}(\alpha)\|f\|_{p, \phi}\|a\|_{q, \psi}, \quad \text { i.e. } \quad L=\|f\|_{p, \phi}^{p-1}<k_{\lambda_{1}}(\alpha)\|a\|_{q, \psi} .
$$

Hence (34) is valid, which is equivalent to (32). It follows that (32), (33), and (34) are equivalent.

If there exists a positive number $k \leq k_{\lambda_{1}}(\alpha)$, such that (32) is still valid as we replace $k_{\lambda_{1}}(\alpha)$, by $k$, then in particular, (20) is valid $\left(\tilde{a}_{n}, \tilde{f}(x)\right.$ are taken as (19)). Then we have (21). For $\varepsilon \rightarrow 0^{+}$in (21), we have $k \geq \frac{1}{\alpha} B\left(\lambda_{2}, \lambda_{1}\right)=k_{\lambda_{1}}(\alpha)$. Hence, $k=k_{\lambda_{1}}(\alpha)$ is the best value of (32). We conform that the constant factor $k_{\lambda_{1}}(\alpha)$ in (33) [(34)] is the best possible, otherwise we can get a contradiction by (35) [(39)] that the constant factor in (32) is not the best possible.

Remark 1 (i) Define a half-discrete Hilbert's operator $T: L_{p, \phi}(\gamma, \infty) \rightarrow l_{p, \psi^{1-p}}$ as follows: For $f \in L_{p, \varphi}(\gamma, \infty)$, we define $T f \in l_{p, \psi^{1-p}}$, satisfying

$$
\operatorname{Tf}(n)=\int_{\gamma}^{\infty} \frac{f(x)}{\left[(x-\gamma)^{\alpha}+(n-\beta)^{\alpha}\right]^{\lambda}} d x \quad(n \in \mathbf{N}) .
$$


Then by (33), it follows $\|T f\|_{p, \psi^{1-p}} \leq k_{\lambda_{1}}(\alpha)\|f\|_{p, \phi}$, i.e. $T$ is the bounded operator with $\|T\| \leq k_{\lambda_{1}}(\alpha)$. Since the constant factor $k_{\lambda_{1}}(\alpha)$ in (33) is the best possible, we have $\|T\|=k_{\lambda_{1}}(\alpha)$.

(ii) Define a half-discrete Hilbert's operator $\tilde{T}: l_{q, \psi} \rightarrow L_{q, \phi^{1-q}}(\gamma, \infty)$ in the following way: For $a \in l_{q, \psi}$, we define $\tilde{T} a \in L_{q, \phi^{1-q}}$, satisfying

$$
\tilde{T} a(x)=\sum_{n=1}^{\infty} \frac{a_{n}}{\left[(x-\gamma)^{\alpha}+(n-\beta)^{\alpha}\right]^{\lambda}} \quad(x \in(\gamma, \infty)) .
$$

Then by (34), it follows $\|\tilde{T} a\|_{q, \phi^{1-q}} \leq k_{\lambda_{1}}(\alpha)\|a\|_{q, \psi^{\prime}}$, i.e. $\tilde{T}$ is the bounded operator with $\|\tilde{T}\| \leq k_{\lambda_{1}}(\alpha)$. Since the constant factor $k_{\lambda_{1}}(\alpha)$ in (34) is the best possible, we have $\|\tilde{T}\|=k_{\lambda_{1}}(\alpha)$.

Theorem 2 Suppose that $0<p<1, \frac{1}{p}+\frac{1}{q}=1,0<\alpha \leq 1,0 \leq \beta \leq \frac{1}{2}, \gamma \in(-\infty,+\infty)$, $\lambda_{1}>0,0<\lambda_{2} \alpha \leq 1, \lambda=\lambda_{1}+\lambda_{2}, \quad \psi(n)=(n-\beta)^{q\left(1-\lambda_{2} \alpha\right)-1}$, $\tilde{\phi}(x)=\left(1-\theta_{\lambda}(x)\right)(x-\gamma)^{p\left(1-\lambda_{1} \alpha\right)-1}\left(\theta_{\lambda}(x)=\frac{1}{B\left(\lambda_{1}, \lambda_{2}\right)} \int_{0}^{\left(\frac{1-\beta}{x-\gamma}\right)^{\alpha}} \frac{u^{\lambda_{2}-1}}{(1+u)^{\lambda}} d u \in(0,1)\right), f(x), a_{n} \geq$ 0 , such that $f \in L_{p, \tilde{\phi}}(\gamma, \infty), a=\left\{a_{n}\right\}_{n=1}^{\infty} \in l_{q, \psi},\|f\|_{p, \tilde{\phi}}>0,\|a\|_{q, \psi}>0$, then we have the following equivalent inequalities:

$$
\begin{aligned}
I & =\sum_{n=1}^{\infty} a_{n} \int_{\gamma}^{\infty} \frac{f(x)}{\left[(x-\gamma)^{\alpha}+(n-\beta)^{\alpha}\right]^{\lambda}} d x \\
& =\int_{\gamma}^{\infty} f(x) \sum_{n=1}^{\infty} \frac{a_{n} d x}{\left[(x-\gamma)^{\alpha}+(n-\beta)^{\alpha}\right]^{\lambda}}>k_{\lambda_{1}}(\alpha)\|f\|_{p, \tilde{\phi}}\|a\|_{q, \psi^{\prime}} \\
J & =\left\{\sum_{n=1}^{\infty}(n-\beta)^{p \lambda_{2} \alpha-1}\left[\int_{\gamma}^{\infty} \frac{f(x)}{\left[(x-\gamma)^{\alpha}+(n-\beta)^{\alpha}\right]^{\lambda}} d x\right]^{p}\right\}^{1 / p} \\
& >k_{\lambda_{1}}(\alpha)\|f\|_{p, \tilde{\phi}^{\prime}} \\
\tilde{L} & :=\left\{\int_{\gamma}^{\infty} \frac{(x-\gamma)^{q \lambda_{1} \alpha-1}}{\left[1-\theta_{\lambda}(x)\right]^{q-1}}\left[\sum_{n=1}^{\infty} \frac{a_{n}}{\left[(x-\gamma)^{\alpha}+(n-\beta)^{\alpha}\right]^{\lambda}}\right]^{q} d x\right\}^{1 / q} \\
& >k_{\lambda_{1}}(\alpha)\|a\|_{q, \psi}
\end{aligned}
$$

where the constant factor $k_{\lambda_{1}}(\alpha)=\frac{1}{\alpha} B\left(\lambda_{1}, \lambda_{2}\right)$ is the best possible.

Proof. By (9) and the reverse of (13) and $0<\|f\|_{p, \tilde{\phi}}<\infty$, we have (43). Using the reverse Hölder's inequality, we obtain the reverse form of (36) as follows

$$
I \geq J\|a\|_{q, \psi} .
$$

Then by (43), (42) is valid. 
On the other hand, if (42) is valid, setting $a_{n}$ as (36), then (37) still holds with $0<p<1$. By (42), it follows that $J>0$. If $J=\infty$, then (43) is trivially valid; if $J<\infty$, then $0<\|a\|_{q, \psi}=J^{p-1}<\infty$, and we have

$$
\|a\|_{q, \psi}^{q}=J^{p}=I>k_{\lambda_{1}}(\alpha)\|f\|_{p, \tilde{\phi}}\|a\|_{q, \psi}, \quad \text { i.e. } \quad J=\|a\|_{, \psi}^{q-1}>k_{\lambda_{1}}(\alpha)\|f\|_{\tilde{\phi}^{\prime}}
$$

Hence (43) is valid, which is equivalent to (42).

By the reverse of (14), in view of $\varpi(x)>k_{\lambda_{1}}(\alpha)\left(1-\theta_{\lambda}(x)\right)$ and $q<0$, we have

$$
\tilde{L}>k_{\lambda_{1}}^{\frac{q-1}{q}} L_{1} \geq k_{\lambda_{1}}^{\frac{q-1}{q}}(\alpha)\left\{k_{\lambda_{1}}(\alpha) \sum_{n=1}^{\infty}(n-\beta)^{q\left(1-\lambda_{2} \alpha\right)-1} a_{n}^{q}\right\}^{1 / q}=k_{\lambda_{1}}(\alpha)\|a\|_{q, \psi},
$$

then (44) is valid. By the reverse Hölder's inequality again, we have

$$
\begin{aligned}
& I= \int_{\gamma}^{\infty}\left[\frac{(x-\gamma)^{\gamma 1 \alpha-\frac{1}{q}}}{\left(1-\theta_{\lambda}(x)\right)^{\frac{1}{q}}} \sum_{n=1}^{\infty} \frac{a_{n}}{\left[(x-\gamma)^{\alpha}+(n-\beta)^{\alpha}\right]^{\lambda}}\right] \\
& \times\left[\left(1-\theta_{\lambda}(x)\right)^{\frac{1}{p}}(x-\gamma)^{\frac{1}{q}-\lambda_{1} \alpha} f(x)\right] d x \\
& \geq \tilde{L}\|f\|_{p, \tilde{\phi}}
\end{aligned}
$$

Hence (42) is valid by (44). On the other hand, if (42) is valid, setting

$$
f(x)=\frac{(x-\gamma)^{q \lambda_{1} \alpha-1}}{\left[1-\theta_{\lambda}(x)\right]^{q-1}}\left[\sum_{n=1}^{\infty} \frac{a_{n}}{\left[(x-\gamma)^{\alpha}+(n-\beta)^{\alpha}\right]^{\lambda}}\right]^{q-1} \quad(x \in(\gamma, \infty)),
$$

then $\|f\|_{p, \tilde{\phi}}^{p}=\int_{\gamma}^{\infty}\left[1-\theta_{\lambda}(x)\right](x-\gamma)^{p\left(1-\lambda_{1} \alpha\right)-1} f^{p}(x) d x=\tilde{L}^{q}=I$. By the reverse of (14), it follows that $\tilde{L}>0$. If $\tilde{L}=\infty$, then (44) is trivially valid; if $0<\tilde{L}<\infty$, then by (42), we have

$$
\|f\|_{p, \tilde{\phi}}^{p}=\tilde{L}^{q}=I>k_{\lambda_{1}}(\alpha)\|f\|_{p, \tilde{\phi}}\|a\|_{q, \psi}, \quad \text { i.e. } \quad \tilde{L}_{r}=\|f\|_{p, \tilde{\phi}}^{p-1}>k_{\lambda_{1}}(\alpha)\|a\|_{q, \psi} .
$$

Hence (44) is valid, which is equivalent to (42). It follows that (42), (43), and (44) are equivalent.

If there exists a positive number $k \geq k_{\lambda_{1}}(\alpha)$, such that (42) is still valid as we replace $k_{\lambda_{1}}(\alpha)$ by $k$, then in particular, (22) is valid. Hence we have (23). For $\varepsilon \rightarrow 0^{+}$ in (23), we obtain $k \leq \frac{1}{\alpha} B\left(\lambda_{1}, \lambda_{2}\right)=k_{\lambda_{1}}(\alpha)$. Hence $k=k_{\lambda_{1}}(\alpha)$ is the best value of (42). We conform that the constant factor $k_{\lambda_{1}}(\alpha)$ in (43) [(44)] is the best possible, otherwise we can get a contradiction by (45) [(46)] that the constant factor in (42) is not the best possible.

In the same way, for $p<0$, we also have the following result:

Theorem 3 If the assumption of $p>1$ in Theorem 1 is replaced by $p<0$, then the reverses of (32), (33), and (34) are valid and equivalent. Moreover, the same constant factor is the best possible.

Remark 2 (i) For $\beta=\gamma=0, \lambda_{1}=\frac{1}{q \alpha}, \lambda_{2}=\frac{1}{p \alpha}$ in (32), it follows

$$
\sum_{n=1}^{\infty} a_{n} \int_{0}^{\infty} \frac{f(x)}{\left(x^{\alpha}+n^{\alpha}\right)^{1 / \alpha}} d x<\frac{1}{\alpha} B\left(\frac{1}{q^{\alpha}}, \frac{1}{p^{\alpha}}\right)\left\{\int_{0}^{\infty} f^{p}(x) d x\right\}^{1 / p}\left\{\sum_{n=1}^{\infty} a_{n}^{q}\right\}^{1 / q} .
$$


In particular, for $\alpha=1, p=q=2$, (47) reduces to (4). (ii) For $\lambda=\alpha=1, \lambda_{1}=\frac{1}{q}$, $\lambda_{2}=\frac{1}{p}$ in (32), it follows

$$
\sum_{n=1}^{\infty} a_{n} \int_{\gamma}^{\infty} \frac{f(x)}{x+n-\gamma-\beta} d x<\frac{\pi}{\sin (\pi / p)}\left\{\int_{\gamma}^{\infty} f^{p}(x) d x\right\}^{1 / p}\left\{\sum_{n=1}^{\infty} a_{n}^{q}\right\}^{1 / q} .
$$

In particular, for $\gamma=-\frac{1}{2}, \beta=\frac{1}{2}, p=q=2$ in (48), we obtain (5). Hence, inequality (32) is the best extension of (4) and (5) with parameters.

\section{Acknowledgements}

This study was supported by the Emphases Natural Science Foundation of Guangdong Institution, Higher Learning, College and University (No. 05Z026), and Guangdong Natural Science Foundation (No. 7004344).

\section{Authors' contributions}

QH carried out the study, and wrote the manuscript. BY participated in the design of the study, and reformed the manuscript. All authors read and approved the final manuscript.

\section{Competing interests}

The authors declare that they have no competing interests.

Received: 8 March 2012 Accepted: 8 May 2012 Published: 8 May 2012

\section{References}

1. Weyl, H: Singulare integral gleichungen mit besonderer berucksichtigung des fourierschen integral theorems. Inaugeral Dissertation, Gottingen. (1908)

2. Schur, I: Bernerkungen sur Theorie der beschrankten Bilinearformen mit unendlich vielen veranderlichen. J Math. 140, $1-28(1911)$

3. Hardy, GH, Littlewood, JE, Pólya, G: Inequalities. Cambridge University Press, Cambridge (1934)

4. Mitrinović, DS, Pečarić, JE, Fink, AM: Inequalities Involving Functions and Their Integrals and Derivatives. Kluwer Acaremic Publishers, Boston (1991)

5. Yang, B: On Hilbert's integral inequality. J Math Anal Appl. 220, 778-785 (1998). doi:10.1006/jmaa.1997.5877

6. Yang, B, Debnath, L: On the extended Hardy-Hilbert's inequality. J Math Anal Appl. 272, 187-199 (2002). doi:10.1016/ S0022-247X(02)00151-8

7. Jin, J, Debnath, L: On a Hilbert-type linear series operator and its applications. J Math Anal Appl. 371, 691-704 (2010). doi:10.1016/j.jmaa.2010.06.002

8. Yang, B, Rassias, T: On a new extension of Hilbert's inequality. Math Ineq Appl. 8(4), 575-582 (2005)

9. Krnić, M, Pečarić, J: Hilbert's inequalities and their reverses. Publ Math Debrecen. 67(3-4), 315-331 (2005)

10. Azar, L: On some extensions of Hardy-Hilbert's inequality and applications. J Inequal Appl 2009 (2009). Article ID 546829

11. Li, Y, He, B: On inequalities of Hilbert's type. Bull Aust Math Soc. 76, 1-13 (2007). doi:10.1017/50004972700039423

12. Zhong, W: The Hilbert-type integral inequality with a homogeneous kernel of lambda-degree. J Inequal Appl 2008 (2008). Article ID 917392

13. Huang, Q, Yang, B: On a multiple Hilbert-type integral operator and applications. J Inequal Appl 2009 (2009). Article ID 192197

14. Huang, Q: On a multiple Hilbert's inequality with parameters. J Inequal Appl 2010 (2010). Article ID 309319

15. Yang, B: The Norm of Operator and Hilbert-Type Inequalities. Science Press, Beijing (2009) in Chinese

16. Yang, B: Hilbert-Type Integral Inequalities. Bentham Science Publishers Ltd., Dubai (2009)

17. Yang, B: Discrete Hilbert-Type Inequalities. Bentham Science Publishers Ltd., Dubai (2011)

18. Kuang, J: Applied Inequalities. Shangdong Science Technic Press, Jinan (2010) in Chinese

19. Yang, B: A mixed Hilbert-type inequality with a best constant factor. Int J Pure Appl Math. 20(3), 319-328 (2005)

20. Yang, B: A half-discrete Hilbert's inequality. J Guangdong Univ Educ 31(3), 1-8 (2011). in Chinese

21. Yang, B: A half-discrete reverse Hilbert-type inequality with a homogeneous kernel of positive degree. J Zhanjiang Normal College 32(3), 5-9 (2011). in Chinese

22. Yang, B: On a half-discrete Hilbert-type inequality. J Shantou Univ (Natural Science) 26(4), 5-10 (2011). in Chinese

23. Kuang, J: Real and Functional Analysis. Higher Education Press, Beijing (2002) in Chinese

doi:10.1186/1029-242X-2012-106

Cite this article as: Huang and Yang: On a more accurate half-discrete Hilbert's inequality. Journal of Inequalities and Applications 2012 2012:106. 